\title{
Prevalence of germ-line mutations in cancer genes among pancreatic cancer patients with a positive family history
}

\author{
Kari G. Chaffee, MS ${ }^{1}$, Ann L. Oberg, PhD ${ }^{1}$, Robert R. McWilliams, MD², Neil Majithia, MD², \\ Brian A. Allen, $\mathrm{MS}^{3}$, John Kidd, $\mathrm{MS}^{3}$, Nanda Singh, $\mathrm{PhD}^{3}$, Anne-Renee Hartman, $\mathrm{MD}^{3}$, \\ Richard J. Wenstrup, $\mathrm{MD}^{3}$ and Gloria M. Petersen, $\mathrm{PhD}^{1}$
}

\begin{abstract}
Purpose: Panel-based genetic testing has identified increasing numbers of patients with pancreatic ductal adenocarcinoma (PDAC) who carry germ-line mutations. However, small sample sizes or number of genes evaluated limit prevalence estimates of these mutations. We estimated prevalence of mutations in PDAC patients with positive family history.

Methods: We sequenced 25 cancer susceptibility genes in lymphocyte DNA from 302 PDAC patients in the Mayo Clinic Biospecimen Resource for Pancreatic Research Registry. Kindreds containing at least two first-degree relatives with PDAC met criteria for familial pancreatic cancer (FPC), while the remaining were familial, but not FPC.
\end{abstract}

Results: Thirty-six patients (12\%) carried at least one deleterious mutation in one of 11 genes. Of FPC patients, 25/185 (14\%) were carriers, while $11 / 117$ (9\%) non-FPC patients with family history were carriers. Deleterious mutations $(n)$ identified in PDAC patients were BRCA2 (11), ATM (8), CDKN2A (4), CHEK2 (4), MUTYH/MYH (3 heterozygotes, not biallelic), BRCA1 (2), and 1 each in BARD1, MSH2, NBN, PALB2, and PMS2. Novel mutations were found in ATM, BARD1, and PMS2.

Conclusion: Multiple susceptibility gene testing in PDAC patients with family history of pancreatic cancer is warranted regardless of FPC status and will inform genetic risk counseling for families.

Genet Med advance online publication 20 July 2017

Key Words: familial pancreatic cancer (FPC); pancreatic ductal adenocarcinoma (PDAC); pathogenic variant (PV); variant of uncertain significance (VUS)

\section{INTRODUCTION}

Pancreatic cancer is a devastating disease and the fourth leading cause of cancer-related death in the United States. The absolute number of new cases and deaths due to pancreatic cancer has increased steadily since $2004,{ }^{1}$ and Rahib et al. ${ }^{2}$ predict that it will be the second most common cause of cancer mortality by 2030 . Approximately $95 \%$ of pancreatic neoplasms are ductal adenocarcinomas (PDAC), which are the most challenging to treat. The rapid progression of pancreatic cancer and its high rate of mortality underlie the importance of improved risk stratification and early detection.

Numerous studies have demonstrated that family history of pancreatic cancer in a first-degree relative is associated with a two- to threefold increased risk of pancreatic cancer. ${ }^{3}$ Previous epidemiologic studies and case reports have demonstrated pancreatic cancer clustering in families. Familial pancreatic cancer (FPC) has been defined as kindreds with at least a pair of first-degree relatives affected with pancreatic adenocarcinoma ${ }^{4}$ and accounts for approximately 5 to $10 \%$ of patients with pancreatic adenocarcinoma. ${ }^{5}$ This standardized definition is now widely used and has facilitated research addressing a variety of questions, including genetic susceptibility.
Increased risk of pancreatic cancer is associated with several inherited syndromes for which the predisposing genes have been identified. The most prominent syndromes are hereditary breast-ovarian cancer syndrome, particularly due to germ-line mutations in $B R C A 2$, and familial atypical mole and melanoma syndrome, due to mutations in CDKN2A. In the most comprehensive analysis to characterize the genetic variation in FPC patients to date, the Pancreatic Cancer Genetic Epidemiology Consortium found that mutations in the hereditary breast-ovarian cancer genes (BRCA1, $B R C A 2$ ) and $C D K N 2 A$ were identified in $7.4 \%$ of FPC probands. ${ }^{6}$ Pancreatic cancer risk is also increased in Lynch syndrome $(M L H 1, M S H 2, P M S 2 \text {, and } M S H 6)^{7-9}$ and hereditary pancreatitis. ${ }^{10}$ In addition, germ-line mutations in $P A L B 2^{6,11,12}$ and $A T M^{7,12,13}$ have been identified among FPC probands using next-generation sequencing (NGS), extending the catalog of predisposing genes. Functional roles for identified germ-line mutations in these two genes were further supported by loss of heterozygosity of the wild-type allele in the pancreatic tumor of the mutation carriers. ${ }^{11,12}$

Pancreatic cancer thus appears to be a genetically heterogeneous disease that is associated with a number of syndromes. Although the American College of Gastroenterology provides some guidance about what genes may be

${ }^{1}$ Department of Health Sciences Research, Mayo Clinic, Rochester, Minnesota, USA; ${ }^{2}$ Department of Oncology, Mayo Clinic, Rochester, Minnesota, USA; ${ }^{3}$ Myriad Genetics Laboratories, Inc., Salt Lake City, Utah, USA. Correspondence: Gloria M. Petersen (petersen.gloria@mayo.edu)

Submitted 23 November 2016; accepted 1 May 2017; advance online publication 20 July 2017. doi:10.1038/gim.2017.85 
appropriate for testing, these guidelines are limited to patients who meet criteria for FPC. ${ }^{14}$ Importantly, germ-line mutations in genes with known pancreatic cancer risk are associated with increased risk of other cancers. Hence, panel testing of multiple cancer-risk genes is likely to increase etiologic information and potentially enhance genetic risk assessment of pancreatic cancer patients who report a positive family history not limited to FPC.

Previously, kindreds treated at the Mayo Clinic that contained at least two affected biological relatives with pancreatic cancer were assessed for mutations in four PDAC-risk genes. ${ }^{6}$ In light of the genetic heterogeneity of pancreatic cancer, here we more comprehensively assessed the prevalence of genetic variants in this cohort. This was done by analyzing 25 cancer susceptibility genes in one affected member per kindred, and included individuals with a personal and family history of pancreatic cancer not limited to FPC. Genes with known pancreatic cancer risk were analyzed, including the hereditary breast-ovarian cancer genes, Lynch syndrome genes, and familial melanoma genes. Additional high- and moderate-penetrance genes associated with increased risk of other cancers, including breast, ovarian, and colorectal, were also included.

\section{MATERIALS AND METHODS}

\section{Patients}

Institutional review board approval was granted at Mayo Clinic, and written consent was obtained from all patients in order to be included in the study. Affected patients were recruited into the Biospecimen Resource for Pancreatic Research registry between the years 2000 and 2013 at the time they sought clinical care at Mayo Clinic. Demographic, clinical, and pathological data are maintained in the registry. A diagnosis of PDAC in affected patients was established via biopsy or clinical documentation. Individuals with hereditary pancreatitis were excluded. Family history information was self-reported in questionnaires, including reports of cancers in family members. Kindreds with at least one pair of firstdegree relatives who were affected with PDAC were considered FPC; kindreds with at least two affected blood relatives that did not meet the FPC definition were considered "familial non-FPC."

Unrelated affected patients in FPC or familial non-FPC kindreds who provided a blood sample were eligible for the study. Although the vast majority of patients were included in our prior report of four genes $(300 / 302,99.3 \%),{ }^{6} 21$ additional genes were tested in this study. This included 16 genes known to be associated with PDAC (APC, ATM, BMPR1A, BRCA1, BRCA2, CDK4, CDKN2A, EPCAM, MLH1, MSH2, MSH6, PALB2, PMS2, SMAD4, STK11, and TP53) and nine genes with no known association with PDAC (BARD1, BRIP1, CDH1, CHEK2, MUTYH/MYH, NBN, PTEN, RAD51C, and $R A D 51 D)$. All genes included in the American College of Gastroenterology guidelines for pancreatic cancer genetic testing are included in this panel. ${ }^{14}$ Each patient's frozen buffy-coat sample was de-identified and labeled with a unique identifier, and all samples were sent to Myriad Genetic Laboratories (Salt Lake City, UT) for DNA extraction and mutation testing.

\section{Next-generation sequencing (NGS) assay}

Sample preparation for NGS was performed from DNA extracted from buffy coats using the RainDance microdroplet polymerase chain reaction system (RainDance Technologies, Billerica, MA). ${ }^{15}$ Briefly, polymerase chain reaction products representing exons and proximal splicing elements of patient DNA were amplified in merged droplets consisting of fragmented patient DNA and select target enrichment primers. These polymerase chain reaction products were subsequently tagged with barcodes and sequencing adaptors for NGS on the Illumina, Inc. (San Diego, CA, USA) HiSeq platform. To circumvent highly homologous pseudogenes, modified sample preparation with long-range and nested polymerase chain reaction followed by NGS on the Illumina MiSeq platform was used for portions of the CHEK2 and PMS2 genes. All clinically actionable variants identified by NGS, and regions that did not meet our preset NGS quality metrics, were independently confirmed with orthogonal sitespecific Sanger sequencing.

To detect exonic deletions and duplications, NGS dosage, microarray comparative genomic hybridization, multiplex ligation-dependent probe amplification, or a combination of these analyses was performed, with all positive results confirmed by an orthogonal method. ${ }^{15}$

\section{Variant classification}

Variants were classified using American College of Medical Genetics and Genomics recommendations, with supporting linkage, biochemical, clinical, functional, and statistical data used for specific missense and intronic alterations. ${ }^{16,17}$ Pathogenic variants (PVs) are those that received a laboratory classification of deleterious or suspected deleterious. Variants for which the pathogenicity could not be determined were categorized as variants of uncertain significance (VUSs). Novel, previously unreported mutations discovered in this study were defined as those not previously identified by the testing laboratory (Myriad Genetic Laboratories, Inc.).

\section{Data analysis}

Analytic methods used in this study have been previously described. ${ }^{6}$ Briefly, prevalence of PV and VUS of the 25 genes studied were compared between individuals of FPC and familial non-FPC kindreds using odds ratios (OR) and 95\% confidence intervals (CI). Descriptive statistics and mutation rates were calculated. Comparisons of the mutation prevalence between groups were measured using either chisquare or Fisher's exact tests, depending on sample sizes. Differences in family history of other cancers by mutation carrier status were quantified using chi-square or Fisher's exact tests. Statistical analyses were completed using SAS 9.4 (SAS Institute, Cary, NC). 
Table 1 Clinical and demographic characteristics, total and by FPC status

\begin{tabular}{|c|c|c|c|c|c|c|}
\hline \multirow{3}{*}{ Median age at PDAC (range) } & \multicolumn{2}{|c|}{ Total $(n=302)$} & \multicolumn{2}{|c|}{ FPC $(n=185)$} & \multicolumn{2}{|c|}{ Familial non-FPC $(n=117)$} \\
\hline & \multicolumn{2}{|c|}{65 (37-93) } & \multicolumn{2}{|c|}{$66.5(37-93)$} & \multicolumn{2}{|c|}{$64(38-87)$} \\
\hline & $N$ & $\%$ & $N$ & $\%$ & $N$ & $\%$ \\
\hline Sex, male & 162 & 53.6 & 98 & 53 & 64 & 54.7 \\
\hline White & 293 & 97.7 & 180 & 98.4 & 113 & 96.6 \\
\hline African American & 4 & 1.3 & 3 & 1.6 & 1 & 0.9 \\
\hline Multiracial & 1 & 0.3 & 0 & 0 & 1 & 0.9 \\
\hline Missing & 2 & - & 2 & - & 0 & - \\
\hline \multicolumn{7}{|l|}{ Ethnicity } \\
\hline Non-Hispanic/non-Latino & 291 & 99.7 & 176 & 100 & 115 & 99.1 \\
\hline Hispanic/Latino & 1 & 0.3 & 0 & 0 & 1 & 0.9 \\
\hline Yes & 8 & 2.7 & 8 & 4.4 & 0 & 0 \\
\hline Missing & 6 & - & 4 & - & 2 & - \\
\hline \multicolumn{7}{|l|}{ Number PDAC per kindred } \\
\hline 2 & 239 & 79.2 & 128 & 69.2 & 111 & 94.9 \\
\hline 3 & 44 & 14.6 & 38 & 20.5 & 6 & 5.1 \\
\hline$\geq 4$ & 19 & 6.2 & 19 & 10.2 & 0 & 0 \\
\hline \multicolumn{7}{|l|}{ PDAC stage } \\
\hline Resectable & 88 & 31.7 & 48 & 29.1 & 40 & 35.4 \\
\hline Locally advanced & 77 & 27.7 & 46 & 27.9 & 31 & 27.4 \\
\hline Metastatic & 113 & 40.6 & 71 & 43 & 42 & 37.2 \\
\hline
\end{tabular}

FPC, familial pancreatic cancer; PDAC, pancreatic ductal adenocarcinoma.

\section{RESULTS}

Patient characteristics and variant results of the 302 study participants are presented in Table 1; 185 (61.3\%) were FPC, and 117 (38.7\%) were familial non-FPC. Median age of diagnosis was 65 years (range 37-93 years), and 53.6\% were males. The tested patients were almost entirely white/ Caucasian (97.7\%), and 8 (2.7\%) were of Ashkenazi Jewish descent. The distribution of pancreatic cancer stage was similar to what would be expected in an unselected pancreatic cancer patient population, with roughly equal proportions of resectable, locally advanced, and metastatic cancer. There were no substantial differences between the two familial groups. Most kindreds (79.2\%) contained two affected members with pancreatic cancer, $14.6 \%$ reported three affected individuals, and $6.2 \%$ contained four or more affected individuals. Patients in the FPC group reported a higher number of PDAC per kindred relative to the familial nonFPC group.

Table 2 summarizes germ-line PV prevalence among the 302 tested patients. The aggregate prevalence was 36/302 (11.9\%) for all cases with any positive PDAC family history. When results were stratified by familial group, the probability that any mutation was identified in an FPC patient was 13.5\%, compared to $9.4 \%$ among familial non-FPC patients $(\mathrm{OR}=1.5 ; 95 \% \mathrm{CI}=0.7-3.2 ; P=0.28)$.

The distribution of PVs is presented in Figure 1. PVs were identified in 11 genes, including seven PDAC-associated genes (ATM, BRCA1, BRCA2, CDKN2A, MSH2, PALB2, and $P M S 2)$ and four genes with no known PDAC association (BARD1, CHEK2, MUTYH/MUY, NBN). Overall, 75\% of PVs detected were in genes known to be associated with PDAC. There was a statistically significant higher proportion of PVs in PDAC-associated genes in the FPC group (11.9\%) compared to the non-FPC group (4.3\%) (OR $=3.0 ; 95 \%$ $\mathrm{CI}=1.1-8.2 ; P=0.02)$. There were no statistically significant differences in the PV positive rate for genes with no known PDAC association between the FPC and non-FPC groups $(1.6 \%$ vs. $5.1 \% ; P=0.09)$, although the number of PVs was small.

Twenty-seven individuals were found to carry a PV in a gene associated with increased risk of breast cancer ( $A T M$, BARD1, BRCA1, BRCA2, CHEK2, PALB2, NBN). A larger proportion of breast cancer PV carriers had a first-degree relative with breast cancer $(13 / 27,48.1 \%)$ versus noncarrier patients $(75 / 275$ (27.3\%); $P=0.02)$. When limited to only female patients, the proportions were $9 / 12(75 \%)$ for breast 
Table 2 Germ-line PV prevalence by type of familial patient (patients meeting FPC criteria versus familial non-FPC), categorized by genes associated and not associated with PDAC

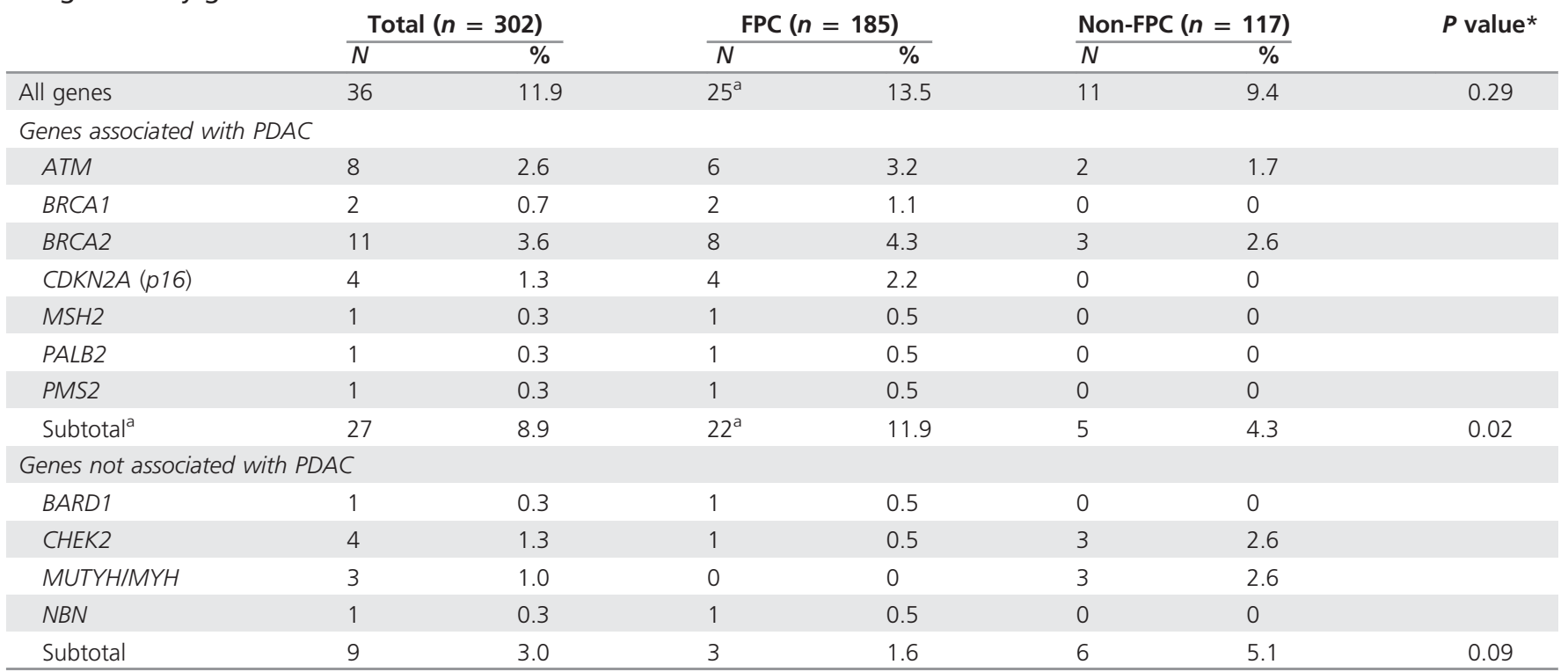

FPC, familial pancreatic cancer; PDAC, pancreatic ductal adenocarcinoma; PV, pathogenic variants.

Genes with no PV included APC, BMPR1A, BRIP1, CDH1, CDK4, EPCAM, MLH1, MSH6, PTEN, RAD51C, RAD51D, SMAD4, STK11, and TP53.

${ }^{a}$ One FPC case carried two PVs: one in BRCA1 and one in BRCA2.

${ }^{*} P$ value compares prevalence of mutations in FPC versus familial non-FPC probands.

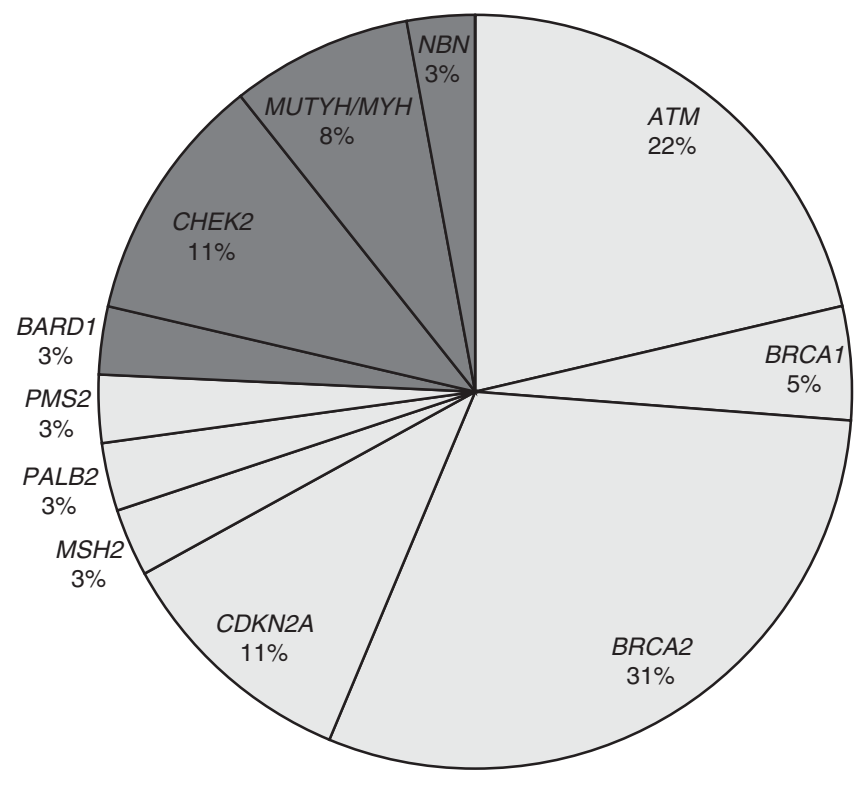

Figure 1 Distribution of pathogenic variants in 302 patients with pancreatic ductal adenocarcinoma (PDAC) with positive family history. Genes associated with PDAC are colored in light gray; genes not known to be associated with PDAC are colored in dark gray.

cancer PV carriers and 38/128 (29.7\%) for noncarriers $(P=0.003)$.

Table 3 lists patient characteristics of the 36 patients who tested positive for at least one germ-line PV. All but one patient who tested positive for a PV carried a mutation in only one tested gene. The single exception was an FPC patient of Ashkenazi Jewish heritage who carried mutations in both BRCA1 (187delAG) and BRCA2 (6174delT); this double mutation carrier has been previously reported. ${ }^{6}$

Supplementary Table 1 summarizes the prevalence of VUS by patient's family history status. The aggregate prevalence is $90 / 302(29.8 \%)$ for all cases with any positive family history. The probability that a patient carried a VUS in any gene was $31.4 \%$ and $27.4 \%$ for FPC versus familial non-FPC, respectively; these rates were not statistically significantly different. There were no statistically significant differences in the PDAC-associated or non-PDAC-associated VUS rates by family status.

Supplementary Table 2 summarizes the PVs and VUS in the 25 genes for this sample; novel variants are highlighted. The five novel PVs detected were ATM c.741dup (p. Arg248Serfs ${ }^{\star}$ ); ATM c.1978del (p.Met660Trpfs $\left.{ }^{\star} 4\right) ; A T M$ c.8264dup (p.Tyr2755*); BARD1 c.632T >A (p.Leu211*); and PMS2 c.2175-1G > A, IVS12-1G >A. The seven novel VUS detected were APC c.4462T $>$ G (p.Leu1488Val); CDH1 c.258A > C (p.Lys86Asn); CDKN2A c.329del (p.Gly110Valfs ${ }^{\star 62}$ ); $\quad$ NBN $\quad$ c.1490C $>$ T $\quad$ (p.Thr497Ile); PMS2 c.2453T > C (p.Ile818Thr); RAD51C c.1103G > A (p.Arg368Gln); and RAD51D c.287G > T (p.Gly96Val).

\section{DISCUSSION}

In this study, we tested newly extracted lymphocyte DNA from 302 pancreatic cancer patients who reported a positive family history using a genetic testing panel of 25 cancer predisposition genes. We found $11.9 \%$ of patients carried a 

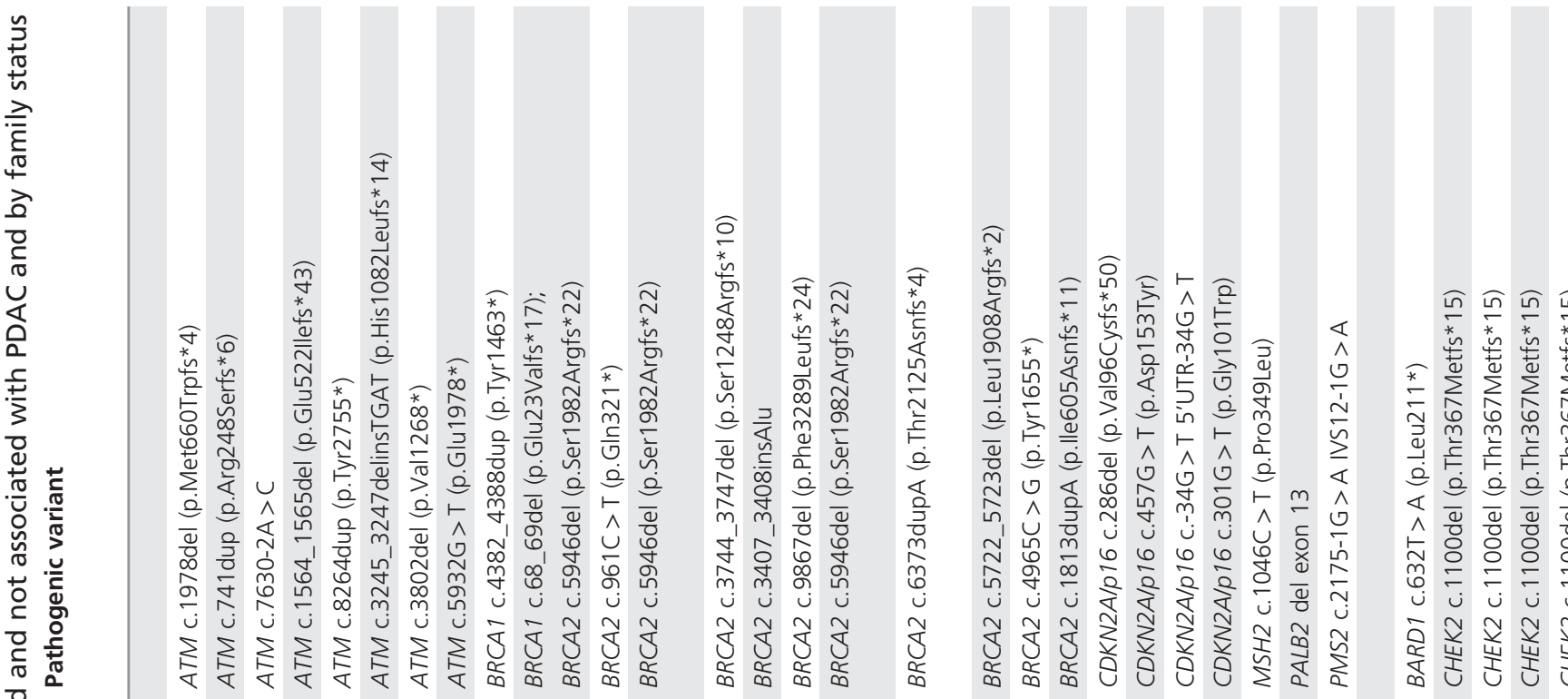

竞

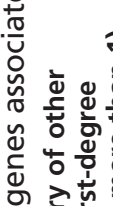

बा $\geq$

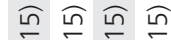
$\widehat{x}$ $-\leqslant \leqslant$

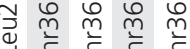

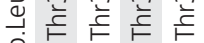

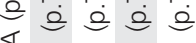
$\wedge$ ब $\overline{\frac{\alpha}{0}} \overline{\frac{\sigma}{0}} \overline{\frac{\alpha}{0}}$

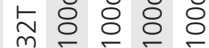
نِّ 穴

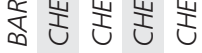

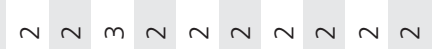

운 운 운 운

눈

눈 운 운

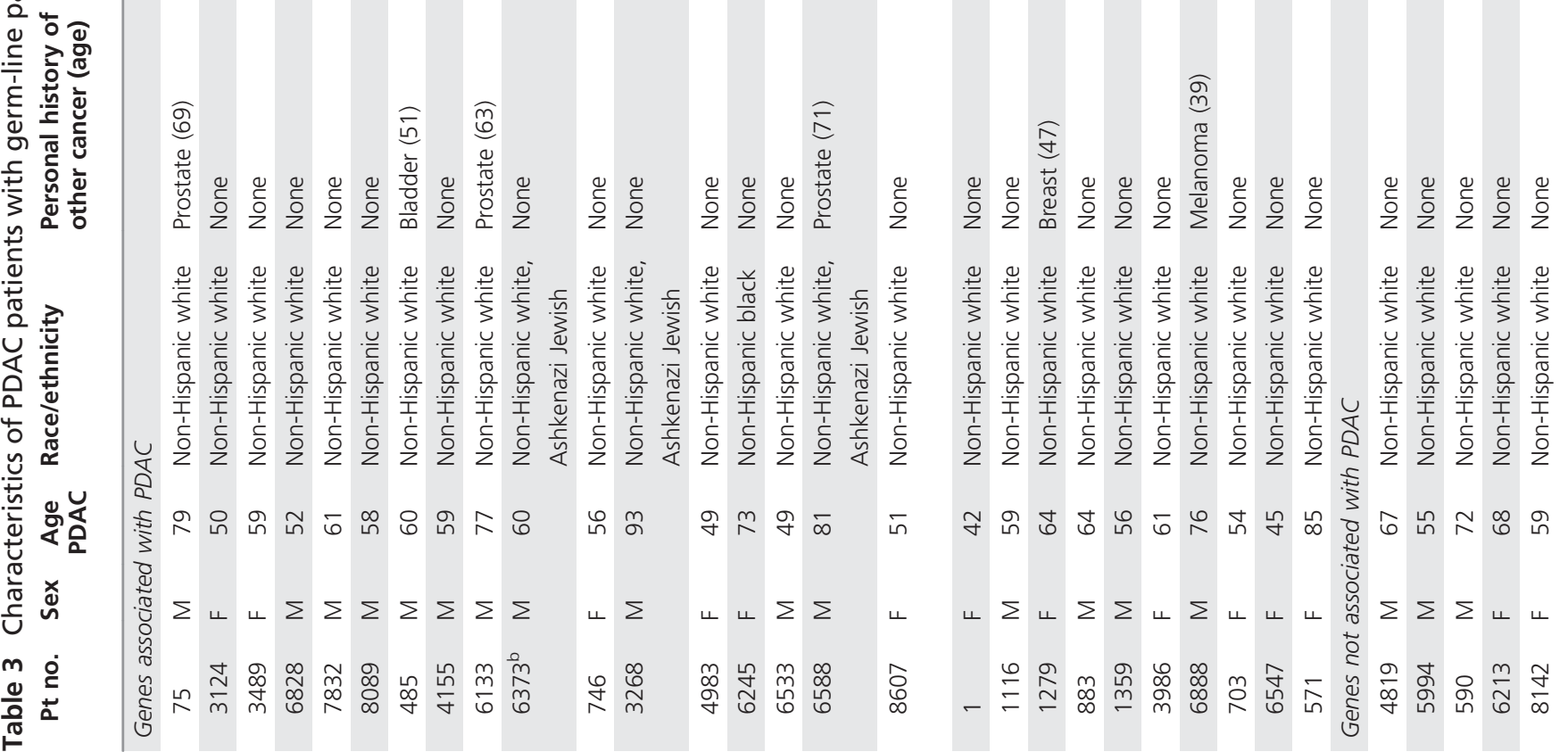




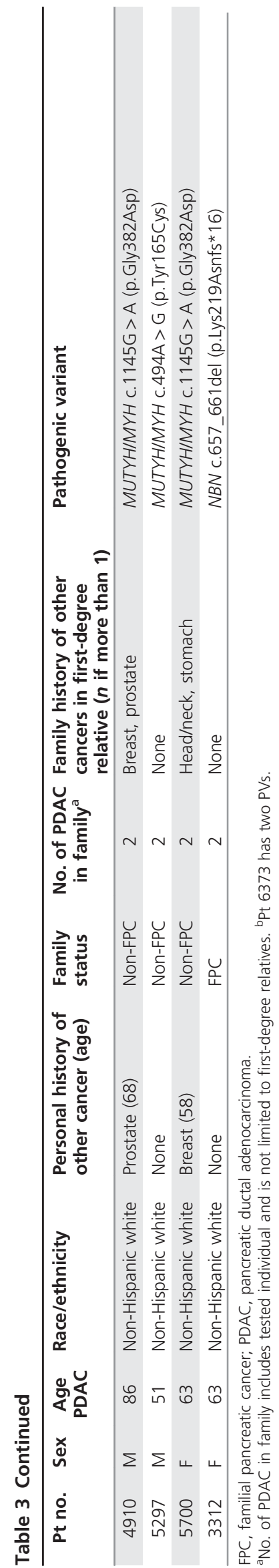

PV in at least one of 11 genes included on the panel. The majority of PVs were in genes previously known to be associated with pancreatic cancer (ATM, BRCA1, BRCA2, CDKN2A, MSH2, PALB2, and PMS2). We observed mutations in genes not currently included in the catalog of genes associated with pancreatic cancer susceptibility (BARD1, CHEK2, MUTYH/MYH, and NBN). When analyzing by FPC status, we found only a slightly higher overall prevalence of PVs among FPC (13.5\%) patients versus non-FPC patients (9.4\%). However, PDAC-associated variants were analyzed, the prevalence of PV among FPC patients was higher than among non-FPC patients $(11.9 \%$ vs. $4.3 \%)$, which is consistent with our previous finding when only four PDACassociated genes were analyzed. ${ }^{6}$

When results of germ-line genetic mutations in pancreatic cancer across multiple studies are considered, there is the expected observation that more mutations are detected when the number of tested genes increases. We found that the overall mutation prevalence of $11.9 \%$ was higher than the previous report assessing mutations in four genes in this same cohort (10.4\%). ${ }^{6}$ Increasing the gene panel from 4 to 25 genes thus increased the PV identification rate by $1.5 \%$. The combined mutation prevalence of BRCA1 and BRCA2 genes in our current study $(12 / 302,4.0 \%)$, is also similar to that reported by Holter et al. ${ }^{18}$ in an unselected series (14/306, $4.6 \%$ ). However, it could be argued that the incremental difference in the overall positive rate suggests that the detection rate may be saturated even when more genes are interrogated. For example, the overall positive mutation rate here is slightly lower than that reported by $\mathrm{Hu}$ et $\mathrm{al} .{ }^{19}$ In a study of 21 genes in 96 consecutive PDAC cases unselected for family history, $\mathrm{Hu}$ et al. found that $13.5 \%$ of patients were found to carry a pathogenic mutation. It should be noted that two patients included in the $\mathrm{Hu}$ et al. study were also included here, including one BRCA2-positive patient. In another study, Grant et al. ${ }^{7}$ examined 13 genes in 290 PDAC cases and reported a mutation prevalence of only $3.8 \%$ (11/290). This lower mutation prevalence is likely related to the fact that the cohort assessed by Grant et al. was lower risk, with nearly two-thirds of cases reporting no family history of pancreatic, breast, or ovarian cancer.

On the other hand, we also observed PVs in four genes that are not considered to be PDAC-associated. This observation, combined with the discovery of novel PV in ATM, BARD1, and PMS2, and the finding that $80 / 266$ (30.1\%) of patients carried VUS but not PV (and will require further investigation to determine whether they are deleterious or not) opens new areas for investigation. Interestingly, 10/36 (28\%) PV carriers also carried a VUS as well, though not necessarily in the same gene in which the PV occurred (only one ATM PV carrier also had a VUS in that gene; and only two CDKN2A PV carriers also had a VUS in that gene).

Overall, 27 individuals were found to carry mutations in genes associated with an increased risk of breast cancer, the most common of which was BRCA2. Although there was an 


\section{ORIGINAL RESEARCH ARTICLE}

increased family incidence of breast cancer among these individuals, only $48 \%$ of these individuals reported a history of breast cancer in a first-degree relative. However, a previous study by Rosenthal et al. ${ }^{20}$ showed that only $25 \%$ of women tested with a multigene panel who had a mutation in a breast cancer risk gene had a family history consistent with an increased risk of breast cancer. These findings were observed for high-penetrance genes, such as BRCA1 and BRCA2, as well as moderate-penetrance genes. This suggests that family history may not be apparent in all individuals at increased risk. In addition, the PVs identified in these genes represent clinically actionable findings, with gene-specific management guidelines to minimize risk of breast and/or ovarian cancer for patients and their family members. ${ }^{21}$

After BRCA2, ATM was the most common breast cancer risk gene in which PVs were identified. Eight individuals were identified as carrying a PV in ATM, six of whom were FPC. While there is growing consensus in the pancreatic cancer genetics literature that germ-line mutations in ATM can predispose to pancreatic cancer, there are also caveats. ATM is a large gene, and little is known about the genetic epidemiology of ATM with respect to PDAC. ATM localizes to chromosome $11 \mathrm{q}$ and was named for its association with ataxia telangiectasia. ${ }^{22}$ It belongs to the protein family of PI3K-related protein kinases and plays an important role in DNA repair. In 2012, ATM was reported as a predisposition gene for familial pancreatic adenocarcinoma. ${ }^{13}$ Heterozygous, constitutional ATM mutations were identified in wholegenome and whole-exome sequencing performed on two kindreds with FPC. When the analysis was expanded to consider an additional 166 FPC patients, an additional four patients were found to have deleterious mutations of ATM, compared to none in 190 spouse controls $(P=0.046){ }^{13}$ Along with others, ${ }^{7,12}$ our study has found numerous VUS in $A T M$, and this may indicate that these do not represent putative disease-causing variants.

We also identified four individuals with PVs in CHEK2. The CHEK2 gene plays a role in checkpoint response in the setting of DNA damage and mutation of this gene has been associated with elevated breast cancer ${ }^{23}$ and prostate cancer risk. $^{24}$ The protein is, however, found in a wide range of tissues, and there is some suggestion that a mutation may predispose to thyroid and kidney cancer as well. ${ }^{25}$ In the study by $\mathrm{Hu}$ et al. ${ }^{19}$ of unselected PDAC patients, one patient with no family history was found to carry a PV in CHEK2. Here, all four patients were found to carry CHEK2 c.1100del, the common European founder mutation. ${ }^{26}$ Given that the majority of our population reported European ancestry, this may be consistent with the expected population frequency of this mutation. However, in combination with previous reports, further study is warranted to investigate the possible association with PDAC.

Five individuals were found to carry PVs in genes associated with hereditary colorectal cancer. This includes 3/302 (0.99\%) patients who were found to carry a monoallelic mutation in MUTYH/MYH. The human MutY homologue (MUTYH/
$M Y H)$ is a DNA glycosylase that is implicated in base excision repair. Mutations of this gene have been shown to be affiliated with familial colorectal adenoma and carcinoma in a syndrome termed MUTYH-associated polyposis. ${ }^{27}$ Extracolonic manifestations of this gene mutation are still being investigated, but early reports suggest it may predispose to endometrial $^{28}$ and duodenal cancers. ${ }^{29}$ Although this is the first report of a $M U T Y H / M Y H$ germ-line mutation in PDAC, this positive rate may be similar to the population frequency of monoallelic MUTYH/MYH mutations. A previous study by Tung et al. ${ }^{30}$ in women with breast cancer tested with a multigene panel reported a $1.8 \%$ prevalence of monoallelic MUTYH/MYH mutations. In comparison, an incidence of $2.1 \%$ was reported in a study of patients with colorectal cancer, which is associated with monoallelic MUTYH/MYH mutations. ${ }^{31}$ Collectively, this indicates that additional studies would be necessary to determine whether $M U T Y H / M Y H$ is associated with PDAC or if these represent incidental findings. However, these do represent clinically actionable findings, as professional society guidelines recommend increased screening for individuals with no personal or family history of colorectal cancer who carry a monoallelic MUTYH mutation. $^{32}$

We also identified two individuals with PVs in genes associated with Lynch syndrome. One individual was found to carry a PV in PMS2, which is characterized primarily by predisposition to colorectal and endometrial cancer. Another individual was found to carry a PV in $M S H 2$. While mutations of several genes can result in Lynch syndrome, mutation of $\mathrm{MSH} 2$ appears to carry higher risk than other genes for development of cancer at any site. ${ }^{33}$ In addition to colorectal cancer, $\mathrm{MSH} 2$ mutation has been linked to endometrial, ovarian, gastric, biliary tract, and urinary tract malignancies. ${ }^{8}$ Previous studies have also identified mutations in the mismatch repair genes among patients with PDAC. Kastrinos et al. ${ }^{9}$ showed that over $20 \%$ of families with known mutations in the mismatch repair genes reported at least one case of PDAC. In addition, Yang et al. ${ }^{34}$ genotyped Lynch syndrome genes in 66 PDAC patients and identified PVs in PMS2 in 2 patients. Previous studies have also shown that families with $\mathrm{MSH} 2$ mutations do seem to be at increased risk for PDAC. ${ }^{35}$ Collectively, this supports the association of PDAC with Lynch syndrome and demonstrates the value in including these genes in routine PDAC genetic screening.

This study also identified germ-line mutations with limited previous observations, including BARD1 and NBN. BRCA1associated ring domain $(B A R D 1)$ is a protein that complexes with $B R C A 1$ and localizes to regions of DNA damage, and is thought to play a role in the DNA checkpoint response. It has been speculated that BARD1 mutations may contribute to breast and/or ovarian cancer susceptibility. ${ }^{27,28}$ There has also been some limited observation of PVs in BARD1 among patients with PDAC: Smith et al. ${ }^{36}$ observed a PDAC patient with a BARD1 PV and $\mathrm{Hu}$ et al. ${ }^{19}$ found a $\mathrm{PV}$ in a PDAC patient with a positive family history. Heterozygous PVs in NBN are also associated with increased breast 
cancer risk, as well as other malignancies. ${ }^{37,38} \mathrm{Hu}$ et al. ${ }^{19}$ also reported one patient with no family history of PDAC who was found to carry a PV in $N B N$. In combination with the observations reported here, this suggests that these genes may be associated with an increased pancreatic cancer risk in the setting of FPC.

There were some limitations of this study. The cohort included here was almost entirely European. As such, it is unclear whether the findings presented here are applicable to patients of non-European ancestry. In addition, this study was limited to patients with a personal and family history of PDAC. In the absence of a control group, additional studies would be necessary to investigate causality for PVs identified in genes with no current known association with PDAC.

The foregoing discussion underscores the importance of the need for further research to characterize the genetic risk for PDAC and other cancers in families that may be segregating PV. Importantly, this genetic predisposition extends beyond families who meet the definition of FPC. While our study found that family history of cancer is an important factor in risk assessment and selection for genetic testing, PVs were identified across many genes associated with different cancer genetic syndromes. This highlights the potential advantage of multigene panel testing over syndrome-specific sequential testing approaches in PDAC patients. We anticipate that as more PDAC patients are screened by NGS, the challenges for health-care providers will increase, particularly when interpreting results in the absence of family history. Our results underscore the importance of comprehensive family history assessment combined with multigene panel testing to enhance risk assessment among pancreatic cancer patients and their families.

\section{Conclusions}

With $12 \%$ prevalence of deleterious mutations, multiple susceptibility gene testing in PDAC patients with a positive family history of pancreatic cancer is warranted regardless of meeting FPC criteria. These findings will inform genetic risk counseling for family members.

\section{SUPPLEMENTARY MATERIAL}

Supplementary material is linked to the online version of the paper at http://www.nature.com/gim

\section{ACKNOWLEDGMENTS}

Funding support for this study includes National Institutes of Health National Cancer Institute SPORE grant P50 CA102701 and National Cancer Institute grant R01CA97075 for the Pancreatic Cancer Genetic Epidemiology Consortium. The authors thank the patients and their families, Krystal Brown, Ph.D., Lynne Pauley, and study assistants Ryan Wuertz, Bridget Eversman, Sarah Amundson, and Megan Reichmann for their contributions to the study. This work was presented at the American Society of Clinical Oncology annual meeting, Chicago, Illinois, 4 June 2016.

\section{DISCLOSURE}

B.A.A., J.K., N.S., A.-R.H., and R.J.W. are employed by Myriad Genetics Laboratories and received salaries and stock options as compensation at the time the research project was performed. The other authors declare no conflict of interest.

\section{REFERENCES}

1. American Cancer Society. Cancer Facts \& Figures 2013. American Cancer Society: Atlanta, 2013.

2. Rahib L, Smith BD, Aizenberg R, Rosenzweig AB, Fleshman JM, Matrisian LM. Projecting cancer incidence and deaths to 2030: the unexpected burden of thyroid, liver, and pancreas cancers in the United States. Cancer Res. 2014;74:2913-2921.

3. Petersen GM. Familial pancreatic adenocarcinoma. Hematol Oncol Clin N Am. 2015:29:641-653

4. Hruban RH, Petersen GM, Ha PK, Kern SE. Genetics of pancreatic cancer: from genes to families. Surg Oncol Clin N Am. 1998;7:1-23.

5. Petersen GM, de Andrade M, Goggins $M$, et al. Pancreatic Cancer Genetic Epidemiology Consortium. Cancer Epidemiol Biomarkers Prev. 2006;15:704-710

6. Zhen DB, Rabe KG, Gallinger $S$, et al. BRCA1, BRCA2, PALB2, and CDKN2A mutations in familial pancreatic cancer: a PACGENE study. Genet Med. 2015:17:569-577.

7. Grant RC, Selander I, Connor AA, et al. Prevalence of germline mutations in cancer predisposition genes in patients with pancreatic cancer. Gastroenterology. 2015;148:556-564.

8. Lin KM, Shashidharan M, Thorson AG, et al. Cumulative incidence of colorectal and extracolonic cancers in $\mathrm{MLH} 1$ and $\mathrm{MSH} 2$ mutation carriers of hereditary nonpolyposis colorectal cancer. J Gastrointest Surg. 1998:2:67-71

9. Kastrinos F, Mukherjee B, Tayob N, et al. Risk of pancreatic cancer in families with Lynch syndrome. JAMA 2009;302:1790-1795.

10. Klein AP. Genetic susceptibility to pancreatic cancer. Mol Carcinog 2012;51:14-24.

11. Jones $\mathrm{S}$, Hruban RH, Kamiyama $\mathrm{M}$, et al. Exomic sequencing identifies PALB2 as a pancreatic cancer susceptibility gene. Science. 2009;324:217.

12. Roberts NJ, Norris AL, Petersen GM, et al. Whole genome sequencing defines the genetic heterogeneity of familial pancreatic cancer. Cancer Discov. 2016:6:166-175.

13. Roberts NJ, Jiao Y, Yu J, et al. ATM mutations in patients with hereditary pancreatic cancer. Cancer Discov. 2012;2:41-46.

14. Syngal S, Brand RE, Church JM, Giardiello FM, Hampel HL, Burt RW. ACG clinical guideline: genetic testing and management of hereditary gastrointestinal cancer syndromes. Am J Gastroenterol. 2015;110: 223-262.

15. Judkins $T$, Leclair $B$, Bowles $K$, et al. Development and analytical validation of a 25 -gene next generation sequencing panel that includes the BRCA1 and BRCA2 genes to assess hereditary cancer risk. BMC Cancer. 2015;15:215.

16. Eggington JM, Bowles KR, Moyes $K$, et al. A comprehensive laboratorybased program for classification of variants of uncertain significance in hereditary cancer genes. Clin Genet. 2014;86:229-237.

17. Richards S, Aziz N, Bale $S$, et al. Standards and guidelines for the interpretation of sequence variants: a joint consensus recommendation of the American College of Medical Genetics and Genomics and the Association for Molecular Pathology. Genet Med. 2015;17:405-424.

18. Holter S, Borgida A, Dodd A, et al. Germline BRCA mutations in a large clinic-based cohort of patients with pancreatic adenocarcinoma. J Clin Oncol. 2015:33:3124-3129.

19. Hu C, Hart SN, Bamlet WR, et al. Prevalence of pathogenic mutations in cancer predisposition genes among pancreatic cancer patients. Cancer Epidemiol Biomarkers Prev. 2016;25:207-211.

20. Rosenthal ET, Evans B, Kidd J, et al. Increased identification of candidates for high-risk breast cancer screening through expanded genetic testing. J Am Coll Radiol. 2017;14:561-568.

21. Daly M, Pilarski R, Berry M, et al. Genetic/familial high-risk assessment: breast and ovarian. NCCN Clinical Practice Guidelines in Oncology (version 2.2017). 2016. http://www.nccn.org/professionals/physician_gls/ pdf/genetics_screening.pdf.

22. Gatti RA, Berkel I, Boder E, et al. Localization of an ataxia-telangiectasia gene to chromosome 11q22-23. Nature. 1988;336:577-580. 
23. The CBCC-CC. CHEK2*1100delC and susceptibility to breast cancer: a collaborative analysis involving 10,860 breast cancer cases and 9,065 controls from 10 studies. Am J Hum Genet. 2004;74:1175-1182.

24. Dong $X$, Wang L, Taniguchi K, et al. Mutations in CHEK2 associated with prostate cancer risk. Am J Hum Genet. 2003;72:270-280.

25. Cybulski C, Gorski B, Huzarski T, et al. CHEK2 is a multiorgan cancer susceptibility gene. Am J Hum Genet. 2004;75:1131-1135.

26. Meijers-Heijboer $H$, Wijnen J, Vasen $H$, et al. The CHEK2 1100 delC mutation identifies families with a hereditary breast and colorectal cancer phenotype. Am J Hum Genet. 2003;72:1308-1314.

27. Al-Tassan N, Chmiel NH, Maynard J, et al. Inherited variants of MYH associated with somatic G:C-> T:A mutations in colorectal tumors. Nat Genet. 2002;30:227-232.

28. Barnetson RA, Devlin L, Miller J, et al. Germline mutation prevalence in the base excision repair gene, $\mathrm{MYH}$, in patients with endometrial cancer. Clin Genet. 2007;72:551-555.

29. Nielsen M, Poley JW, Verhoef S, et al. Duodenal carcinoma in MUTYHassociated polyposis. J Clin Pathol. 2006;59:1212-1215.

30. Tung N, Lin NU, Kidd J, et al. Frequency of germline mutations in 25 cancer susceptibility genes in a sequential series of breast cancer patients. J Clin Oncol. 2016;34:1460-1468.

31. Yurgelun $M B$, Allen $B$, Kaldate RR, et al. Identification of a variety of mutations in cancer predisposition genes in patients with suspected Lynch syndrome. Gastroenterology. 2015;149:604-613.
32. Provenzale D, Gupta S, Ahnen DJ, et al. Genetic/familial high-risk assessment: colorectal version 1.2016. Clinical Practice Guidelines in Oncology. J Natl Compr Canc Netw 2016;14:1010-1030.

33. Vasen HF, Stormorken A, Menko FH, et al. MSH2 mutation carriers are at higher risk of cancer than MLH1 mutation carriers: a study of hereditary nonpolyposis colorectal cancer families. J Clin Oncol. 2001;19: 4074-4080.

34. Yang $X R$, Rotunno $M$, Xiao $Y$, et al. Multiple rare variants in high-risk pancreatic cancer-related genes may increase risk for pancreatic cancer in a subset of patients with and without germline CDKN2A mutations. Hum Genet. 2016;135:1241-1249.

35. Lynch HT, Voorhees GJ, Lanspa SJ, McGreevy PS, Lynch JF. Pancreatic carcinoma and hereditary nonpolyposis colorectal cancer: a family study. Br J Cancer. 1985;52:271-273.

36. Smith AL, Alirezaie N, Connor A, et al. Candidate DNA repair susceptibility genes identified by exome sequencing in high-risk pancreatic cancer. Cancer Lett. 2016;370:302-312.

37. Seemanova $E$, Jarolim $P$, Seeman $P$, et al. Cancer risk of heterozygotes with the NBN founder mutation. J Natl Cancer Inst. 2007;99: 1875-1880.

38. Zhang B, Beeghly-Fadiel A, Long J, Zheng W. Genetic variants associated with breast-cancer risk: comprehensive research synopsis, meta-analysis, and epidemiological evidence. Lancet Oncol. 2011;12: 477-488. 